\title{
Die Strategie der Datenerhebung bei Unternehmensstatistiken in den Niederlanden
}

\author{
Robert Göttgens • Ger Snijkers
}

Online veröffentlicht: 21 September 2007

(C) Springer 2007

Zusammenfassung Ende 2003 ist in den Niederlanden ein neues Statistikgesetz (the Act on Statistics Netherlands, ,CBS-wet“) in Kraft getreten. In diesem Gesetz wurde festgelegt, welche Regeln die amtliche Statistik bei der Erhebung, Verwendung und Verbreitung von Daten zu befolgen hat. Ein wesentliches Ziel dieses Gesetzes besteht in der Reduktion der Auskunftslast. Das Statistikgesetz sieht vor, dass das Statistische Amt befugt ist, gesetzlich verankerte Verwaltungs- und Registerdaten für statistische Zwecke zu nutzen. Wenn derartige Daten die benötigte Information nicht enthalten, darf das Statistische Amt Primärerhebungen durchführen. In diesem Aufsatz wird die allgemeine Strategie beschrieben, die das Statistische Amt der Niederlande bei der Datenerhebung verfolgt. Anhand zweier Beispiele amtlicher Statistiken wird die praktische Implementierung dieser Strategie vorgestellt.

Stichwörter Auskunftslast · Strategie der Datenerfassung · Nutzung von Verwaltungs- und Registerdaten

\begin{abstract}
At the end of 2003 the Act on Statistics Netherlands has become effective (the 'CBS-Act'). This Act establishes the rules Statistics Netherlands has to pursue with regard to acquisition, application and dissemination of data. A major goal of this act is the reduction of the response burden. The Statistics Act states that Statistics Netherlands is authorized, for statistical purposes, to use data from statutory required administrations and registers. In case that registers do not provide the required data, Statistics Netherlands is authorized to conduct primary surveys. In this paper, the general strategy is described Statistics Netherlands pursues when survey-
\end{abstract}

R. Göttgens $(\bullet) \cdot$ G. Snijkers

Division of Business Statistics, Statistics Netherlands, P.O. Box 4481, 6401 Heerlen,

The Netherlands

e-mail: RGTS@CBS.nl 
ing data. The practical implementation of this strategy is exemplified by means of two official statistics.

Keywords Response burden · Data collection strategy · Use of data from administrations and registers

JEL classification C 80, C 81

\section{Einführung}

Ende 2003 ist das Gesetz für das Statistische Amt der Niederlande (the Act on Statistics Netherlands, „CBS-wet“) in Kraft getreten. In diesem Gesetz wurde festgelegt, welche Regeln das Statistische Amt bei der Erhebung, Verwendung und Verbreitung von Daten zu befolgen hat.

In diesem Gesetz ist unter anderem festgelegt, dass das Statistische Amt darüber wacht, dass ,... die Datenerfassung so erfolgt, dass die daraus entstehenden Kosten für Unternehmen und Institutionen minimiert werden ... ‘. Weiter bestimmt das Gesetz, dass das Amt dazu befugt ist, gesetzlich verankerte Verwaltungs- und Registerdaten für statistische Zwecke zu nutzen. Falls derartige Daten die benötigte Information nicht enthalten, darf das Statistische Amt Primärerhebungen bei Unternehmungen, Freiberuflern, Institutionen und Rechtspersonen durchführen. Zusätzlich fordert die Politik für den Zeitraum 2003-2007 eine Abbau der Auskunftslast für die Wirtschaft um 25\%. Für die Legislaturperiode 2007-2011 ist eine weitere Reduzierung um 25\% vorgesehen. Diese Forderungen stellen das Statistische Amt vor eine schwierige Aufgabe.

Aus diesem Anlass, aber auch aus innerem Antrieb, eine effiziente und effektive Herstellung von Statistiken zu gewährleisten, hat das Statistische Amt der Niederlande eine generelle Strategie der Datenerfassung entwickelt. Der Zweck dieser Strategie ist es, das neue Statistikgesetz zu erfüllen und eine weitere Professionalisierung der Betriebsführung zu erreichen. Damit soll die Grundlage geschaffen werden für eine klare, transparente und eindeutige Argumentation in Sachen Datenerfassung.

Die generelle Strategie der Datenerfassung besteht aus zwei Hauptteilen, denen ein notwendiger vorbereitender Teil vorausgeht. Sie kann also gegliedert werden in die drei Schritte

0. Festlegung der Bedürfnisse bezüglich statistischer Information (inkl. Qualitätsansprüche

1. Erfassung von Sekundärdaten

2. Erfassung von Primärdaten

Die Reihenfolge dieser Schritte ist nicht beliebig, sondern widerspiegelt das, was im Statistikgesetz festgelegt ist. Es ist offensichtlich, dass die beiden Arten der Datenerfassung sorgfältig aufeinander abgestimmt sein müssen. In der Praxis wird bei der Zusammenstellung von Statistiken häufig ein Multi-Quellen (,multi-source ${ }^{\text {) }}$-Design entwickelt, in dem verschiedene Datenquellen miteinander kombiniert wird. 


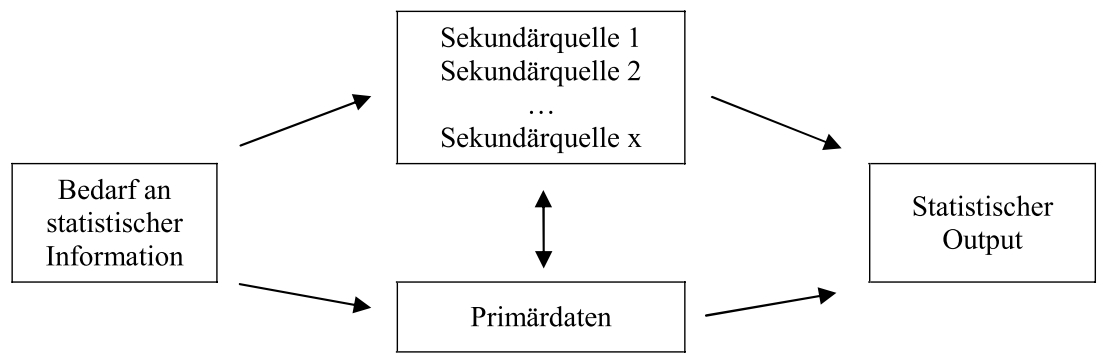

Abb. 1 Multi-Quellen Strategie der Datenerfassung

Das Grundprinzip der niederländischen Strategie ist, dass jeder statistische Output möglichst weitgehend auf Schätzungen beruht oder aus Registerdaten hergeleitet wird. Außerdem müssen Register soweit wie möglich für die statistische Modellentwicklung genutzt werden. Nur wenn dann noch immer ein Teil der benötigten Information fehlt, sei es hinsichtlich der statistischen Variablen oder auch hinsichtlich eines Teils der Population, darf diese Information mittels primärer Datenerfassung erhoben werden.

Neben der kontinuierlichen Erschließung von weiteren Sekundärquellen und dem gleichzeitigen effizienten Einsatz der primären Modi der Datenerfassung liegt die Herausforderung für das Statistische Amt der Niederlande vor allem in der Optimierung der Abstimmung zwischen sekundärer und primärer Datenerfassung.

\section{Die einzelne Schritte der Strategie}

\subsection{Schritt 0 - Festlegung des Informationsbedarfs}

Als Ausgangspunkt für die Strategie der Datenerfassung gilt der Bedarf an statistischer Information. D.h., es ist zu präzisieren, über welche Variablen und welche Populationsparameter Aussagen gemacht werden sollen.

Die Festlegung des statistischen Informationsbedarfs umfasst die nachfolgenden Konkretisierungen:

1. Angabe, auf Grundlage welcher gesetzlichen Regelung, z.B., EU-Verordnung, nationales Gesetz, Vertrag, CCS ${ }^{1}$-Beschluss etc., eine Statistik durch das Statistische Amt realisiert werden muss.

2. Detaillierte Charakterisierung des Informationsbedarfs durch:

- Präzisierung von Konzepten, statistischen Variablen, Outputvariablen, Populationsparametern, etc.;

- Bestimmung der Zielpopulation;

- Präzisierung des Detaillierungsgrades;

\footnotetext{
${ }^{1} \mathrm{CCS}$ : Centrale Commissie voor de Statistiek, Zentrale Statistikkommission
} 
- Festlegung des Untersuchungsrhythmus, etwa monatlich, (viertel)jährlich, oder nur einmalig;

- Fixierung eines Termins, zu dem die statistischen Information vorliegen soll;

- Bestimmung der Genauigkeit der Erfassung;

- Präzisierung weiterer, näher zu spezifizierender Qualitätsforderungen (siehe $\S 2.2)$.

3. Überprüfung, ob die gewünschte Information, eventuell auch teilweise und/oder in anderer Form, schon durch das CBS erfasst wird (zur Vermeidung mehrfacher Datenerfassung, insbesondere Mehrfachbefragung).

4. Im Licht dieser Konkretisierungen erfolgt schließlich:

- die Beurteilung vorhandener Sekundärquellen (siehe $§ 2.2$.) sowie

- die Planung einer primären Datenerfassung (inklusive Entwurf eines Fragebogens, Planung der Stichprobe sowie Organisation der Feldarbeit)

Auf Basis dieser Konkretisierungen und schon existierender Erfahrungen mit vergangenen CBS-Untersuchungen wird eine erste Planung der Untersuchung erarbeitet, inklusive einer ersten Einschätzung der Realisierbarkeit der Untersuchung im Hinblick auf:

- Zeit (realistische Termine und Deadlines)

- Humanressourcen (benötigte Mannjahre)

- finanzielle Mittel (Bereitstellung ausreichender Mittel)

- Qualität (kann die Statistik mit ausreichender Qualität produziert werden).

Im Rahmen dieser Planung wird im Einzelfall eine Pilotstudie erstellt, in der mehrere Szenarien ausgearbeitet sind. Nach Umsetzung dieser Planungsschritte erfolgt auf der Grundlage einer Kosten/Nutzenanalyse ein expliziter Beschluss über Realisierung oder Nichtrealisierung der Untersuchung. Bei der Ausarbeitung der Kosten/Nutzenanalyse werden neben den Kosten der Datenerfassung auch die Kosten der Datenverarbeitung berücksichtigt.

\subsection{Schritt 1 - Erfassung von Sekundärdaten}

Nachdem in Schritt 0 der Informationsbedarf konkretisiert wurde, wird in Schritt 1 der erste konkrete Schritt zur Datenbeschaffung gemacht. Dieser erste Schritt betrifft entsprechend den Vorgaben im Statistikgesetz die Erfassung von Sekundärdaten. Im Beschluss Datenerfassung CBS (,,Besluit gegevensverwerving CBS“) heißt es explizit:

„In erster Instanz muss das CBS benötigte Daten so weit wie möglich aus existierenden Verwaltungen und Registern von Behörden entnehmen. (...) In zweiter Instanz kann das CBS Daten aus Registern von semi-öffentlich-rechtlichen Rechtspersonen benutzen, die in diesem Beschluss aufgeführt werden. (...) Erst in letzter Instanz (...) wird das CBS (...) Unternehmen und Institutionen direkt befragen. Dies geschieht folglich nur, wenn existierende Register (...) den Datenbedarf nicht befriedigen, die benötigten Daten nicht rechtzeitig zur Verfügung stehen oder von unzureichender Qualität sind.“ 
Diese gesetzliche Vorgabe bedeutet, dass sich das CBS auf die Erfassung von Sekundärdaten beschränken muss, falls folgende Kriterien erfüllt werden:

a. Befriedigender Umfang: Daten aus Sekundärquellen sind befriedigend, wenn sie

- die Zielpopulation vollständig erfassen und wenn aus ihnen

- der Informationsbedarf vollständig abgeleitet und/oder geschätzt werden kann.

Im Fall, dass Sekundärquellen den Datenbedarf nur teilweise befriedigen, werden diese uneingeschränkt genutzt. Zugleich wird, häufig nach Rücksprache mit der registerführenden Stelle, nach Möglichkeiten gesucht, um das betreffende Register besser auf den Informationsbedarf auszurichten.

b. Rechtzeitige Verfügbarkeit: die Daten aus Sekundärquellen stehen so zeitig zur Verfügung, dass der Informationsbedarf termingerecht gedeckt werden kann.

c. Hinreichende Qualität: die Sekundärdaten besitzen eine zur Deckung des vorliegenden Informationsbedarfs hinreichende Qualität ${ }^{2}$. Dieses Kriterium lässt offenbar einen gewissen Interpretationsspielraum zu. Generell sollte die Validität der Sekundärdaten untersucht werden. Weiter ist stets zu prüfen, inwieweit die Daten mit Fehlern behaftet sind und vorher gesetzten Qualitätsforderungen gerecht werden (zur Prüfung der Qualität von Registerdaten werden beispielsweise durch Statistics Canada, Statistics Finland und das UK Office for National Statistics (Thomas 2005) Richtlinien ausgearbeitet).

d. Vertretbare Kosten: Grundsätzlich ist die Nutzung von Registern öffentlicher Instanzen für das Amt kostenfrei. Es ist jedoch denkbar, dass die Nutzung einer Sekundärquelle unvertretbar höhere Kosten mit sich mitbringt als primäre Datenerfassung.

Der Schritt 1 der Datenerfassung umfasst folgende Einzelschritte:

1. Es wird untersucht, ob der vorliegende statistische Informationsbedarf mithilfe von Registerdaten geschätzt werden kann. Weiter wird gegebenenfalls geprüft, ob die Registerdaten dadurch nutzbar gemacht werden können, dass die gewünschten Outputvariablen aus den vorhandenen Registervariablen, mit oder ohne Verwendung von Schätzmethoden, hergeleitet werden können. Diese Prüfung findet sowohl für die gesamte Zielpopulation statt als auch Teilmengen davon.

2. Falls sich in Betracht kommende Register zur Deckung des vorliegenden Informationsbedarfs faktisch als nicht oder nur teilweise geeignet erweisen, so wird dies für jedes dieser Register eingehend begründet. Die Notwendigkeit einer solchen Begründung ergibt sich aus dem CBS-Gesetz.

3. Neben den oben genannten Kriterien spielen auch Stabilität und Kontinuität eines Registers eine wichtige Rolle. Wenn das Statistische Amt den Beschluss fasst hat, ein bestimmtes Register zu nutzen, dann muss garantiert werden, dass dieses Register über längere Zeit hinaus genutzt werden kann. Um dies sicherzustellen werden mit der registerführenden Stelle konkrete Vereinbarungen getroffen.

\footnotetext{
${ }^{2}$ Die Nutzung von Sekundärquellen darf nicht zu einer Qualitätsabnahme führen. Es hat sich aber gezeigt, dass die Qualität von Primärdaten häufig wesentlich überschätzt wird (Giesen 2004).
} 


\subsection{Schritt 2 - Erhebung von Primärdaten}

Bei der Durchführung des ersten Schrittes der Datenerfassung kann sich herausstellen, dass zur (vollen) Befriedigung des Informationsbedarfs (zum Teil) eine Erhebung von Primärdaten erforderlich ist. Zur vollen Befriedigung des Informationsbedarfs unbedingt notwendige Primardaten werden in einem zweiten Schritt der Datenerfassung erhoben. Dabei gilt das Prinzip, dass statistische Information, die aus Sekundärdaten geschätzt oder abgeleitet werden kann, nicht mehr primär erhoben wird. Im Ausnahmefall können jedoch folgende Informationen im Rahmen der Erhebung von Primärdaten beschafft werden:

- Variablen, die im primären Prozess als Kovariable, Prozessvariable oder als Nebeninformation erforderlich sind (z.B. um durch einen elektronischen Fragebogen gelotst zu werden, oder als Hilfsvariable für eine Hochrechnung).

- Variablen, die im Fragebogen der Primärerhebung erforderlich sind, um eine zusammenhängende und kohärente Fragenfolge zu erstellen.

- Information über separate Teilpopulationen, wenn die Registerinformation nicht die Gesamtheit der Teilpopulationen deckt.

Für primäre Datenerfassung stehen verschiedene Modi zur Verfügung. Diese sind nach Kosten rangiert die folgenden:

- XBRL (automatisch auszufüllende elektronische Fragebögen)

- Internet (on-line oder off-line selbst auszufüllende elektronische Fragebögen)

- Papier (selbst auszufüllende Fragebögen auf Papier)

- CATI (Computer-Assisted Telephone Interviewing)

- CAPI (Computer-Assisted Personal Interviewing)

Die niederländische Strategie der Datenerfassung geht davon aus, dass bei der Erhebung von Primärdaten zunächst soweit wie machbar möglichst kostengünstige Modi eingesetzt werden. Bei der Wahl des Erhebungsmodus müssen verschiedene Kriterien berücksichtigt werden. Die wichtigsten Kriterien sind die in Schritt 0 festgelegten Qualitätsaspekte (wie Höhe und Selektivität der Rücklaufquoten, Messfehler und Validität), die Geschwindigkeit, mit der die Daten erfasst werden können sowie die anfallenden Kosten. In Tabelle 1 werden einzelne Erfassungsmodi auf einer dreiteiligen Skala evaluiert. Dabei werden diese Aspekte näher differenziert.

Die Bewertung der einzelnen Erfassungsmodi in Tabelle 1 basiert einerseits auf Erfahrungen von Sachverständigen beim Niederländischen Amt, andererseits auf internationalen Erfahrungen (vgl. etwa Czaja und Blair 2005, Groves et al. 2004, Ministerie van de Vlaamse Gemeenschap 2001). In der Literatur wird betont, dass die Wahl eines optimalen Erhebungsmodus nicht in abstracto behandelt werden kann, sondern nur unter Bezugnahme auf ein konkretes Informationsbedürfnis.

Die verschiedenen Erhebungsmodi werden in der Praxis häufig in Kombination eingesetzt, um einen hinreichend umfangreichen und repräsentativen Respons zu erzielen. Je nach Informationsbedürfnis werden Mixed-mode-Varianten entwickelt, bei deren Zusammenstellung jeweils auf die Vorteile der einzelnen Modi abgestellt wird. In Tabelle 1 ist auch angegeben, unter welchen Aspekten Mixed-mode-Varianten der Datenerfassung (im Allgemeinen) aussichtsreich und erfolgreich sind. 
Tabelle 1 Evaluation verschiedener Erfassungsmodi

\begin{tabular}{|c|c|c|c|c|c|}
\hline \multirow[b]{2}{*}{ Bewertungskriterium } & \multicolumn{5}{|c|}{ Modus } \\
\hline & CAPI & CATI & Papier & Internet & Mixed Mode \\
\hline \multicolumn{6}{|l|}{ Datenqualität } \\
\hline Rahmen der Stichprobe & + & o & + & o & + \\
\hline Rücklaufquote & + & + & o & - & + \\
\hline Möglichkeiten Fragebögen ${ }^{(*)}$ & + & o & - & o & + \\
\hline Länge der Fragebögen & + & o & o & - & o \\
\hline \multicolumn{6}{|l|}{ Geschwindigkeit } \\
\hline Feldarbeit (data capture) & - & + & - & + & o \\
\hline Verarbeitung & + & + & - & + & + \\
\hline \multicolumn{6}{|l|}{ Kosten } \\
\hline Feldarbeit (data capture) & - & o & o & + & + \\
\hline Verarbeitung & + & + & - & + & + \\
\hline
\end{tabular}

(*) Im Aspekt Möglichkeiten Fragebögen sind auch Effekte in Zusammenhang mit dem Interviewereinsatz einkalkuliert.

In Rahmen der Planung einer statistischen Untersuchung muss die Strategie der Datenerfassung sorgfältig ausgearbeitet sein. Dabei müssen die in Tabelle 1 genannten Aspekte im Allgemeinen weiter differenziert werden. Meist müssen und können sie geeignet operationalisiert und sowohl qualitativ als auch quantitativ konkret untermauert werden.

\section{Anwendung der Strategie der Datenerhebung}

Die niederländische Strategie der Datenerhebung stellt ein allgemeines Prinzip dar. Für eine Implementierung dieser Strategie werden zwei Fälle unterschieden: (1) neue statistische Untersuchungen und (2) bestehende statistische Untersuchung. Im Fall einer neuen Untersuchung wendet das Amt seine Strategie uneingeschränkt an. Bei existierenden Untersuchungen soll in einem Plan eine Art Migrationspfad ausgearbeitet werden. Bei der konkreten Ausarbeitung eines solchen Plans kann auf Erfahrungen bei anderen statistischen Ämtern (siehe zum Beispiel Statistics Canada, Statistics Finland und ONS) zurückgegriffen werden. Das Ziel eines Migrationspfades ist eine klare, transparente und effiziente Strategie der Datenerfassung. In diesem Abschnitt soll die niederländische Strategie der Datenerhebung anhand zweier Beispiele aus der Praxis exemplifiziert werden.

\subsection{Structural Business Statistics (,,SBS“)}

Die Structural Business Statistics werden auf der Basis einer EU-Verordnung erstellt. Sie stellen eine Untersuchung auf Basis einer geschichteten Stichprobe dar, wobei nach Größe und wirtschaftlicher Aktivität der Unternehmung geschichtet wird (212 Kernzellen). Der Stichprobenumfang beträgt 87.000 Einheiten, davon 50.000 Unternehmen mit weniger als 10 Arbeitnehmern. Bis einschließlich 2004 wurde zur Datenerhebung ausschließlich ein Papierfragebogen verwendet. Dieser ist mit bis zu 200 Variablen sehr lang und recht kompliziert. Die durch die SBS-Statistik verursach- 
ten Bürokratiekosten betragen etwa 17\% der durch die CBS-Unternehmensstatistiken verursachten Gesamtkosten.

Seit dem Berichtsjahr 2005 wird deshalb teilweise die oben beschriebene Strategie der Datenerfassung angewendet. Zuerst wurde in einer Pilotstudie untersucht, in wiefern es möglich ist, Registerdaten der Steuerbehörden zu nutzen. Dabei stellte sich heraus, dass unter bestimmten Bedingungen bei einem Teil der Kernzellen auf Basis von Mehrwertsteuerdaten Umsatzniveaus geschätzt werden können. Darüber hinaus wurde festgestellt, dass es mithilfe von Registerdaten der Gesellschaftssteuer möglich ist, für einen Teil der Kernzellen Niveauschätzungen sowohl von SBSspezifischen als auch von allgemeinen finanziell-ökonomischen Variablen zu realisieren.

Konkretes Resultat der teilweisen Umstellung auf die neue Strategie der Datenerhebung war, dass bei 29 Kernzellen - alles Unternehmen mit weniger als 10 Arbeitnehmern - die Datenerfassung mittels Registerdaten realisiert werden konnte. Das bedeutet, dass für ein Drittel der Unternehmen in der Stichprobe mit weniger als 10 Arbeitnehmern keine direkte Datenerfassung mehr nötig ist. Dies bedeutet, dass etwa 17.000 Fragebögen weniger ausgefüllt werden müssen.

Parallel zu den Untersuchungen hinsichtlich einer möglichen Nutzung von Registerdaten wurde positiv-kritisch analysiert, inwieweit es möglich ist, die primäre Datenerfassung innerhalb der gesetzlichen Verpflichtungen oder (nationalen) Verträgen oder Vereinbarungen, z.B. mit Unternehmerverbänden, speziell im mittelständischen Bereich, zu reduzieren. Es zeigte sich, dass ein großer Teil der Variablen vom Fragebogen gestrichen werden konnte. Er konnte um ungefähr 23\% verkürzt worden.

Als letzter Schritt der Strategie wurde im Jahr 2005 in einer Pilotstudie untersucht, inwieweit Datenerfassung über Internet-Fragebögen möglich ist. Im März 2006 wurde ca. zehn Prozent der Stichprobe - ungefähr 7.500 Unternehmen - angeboten, statt eines schriftlichen Fragebogens über Internet einen elektronischen Fragebogen auszufüllen. Die Resultate der Pilotstudie waren überzeugend: In Bezug auf Umfang, Geschwindigkeit und Selektivität des Rücklaufs wurden keine Unterschiede festgestellt zwischen der Datenerfassung über elektronische Fragebogen und jener mit Hilfe von Papier-Fragebögen. Die Qualität der erfassten Daten zeigte eine leichte Verbesserung (hinsichtlich Konsistenz) und nur 4 Prozent (!) der befragten Unternehmen haben den Wunsch geäußert, statt eines elektronischen Fragebogen weiter einen Papier-Fragebogen zu erhalten.

Im April 2007 wurde bei der Erhebung der SBS-Statistik für das Berichtsjahr 2006 bei 70.000 Unternehmen ein elektronischer Fragebogen eingesetzt.

\subsection{Statistik der Finanzstrukturen von Unternehmen (,SFO“)}

Diese Statistik wird erstellt auf Basis einer niederländischen Rechtsverordnung. Ihre Ergebnisse sind von größter Relevanz für die Volkswirtschaftliche Gesamtrechnung. Die wichtigsten Charakteristika dieser Statistik für die Berichtsjahre bis einschließlich 1992 waren die folgenden: Alle großen Unternehmen $(\mathrm{N}=3.500)$ mit einem Aktivvermögen von mehr als 5 Millionen Euro wurden vollständig erfasst mithilfe eines Papierfragebogens mit bis zu 450 Variablen. Die kleineren Unternehmen wurden auf Basis einer geschichteten Stichprobe (Stratifikation nach Größe und wirtschaft- 
licher Aktivität) vom Umfang $n=10.000$ erfasst. Dazu wurde ein Papierfragebogen mit 45 Variablen verwendet.

In den Jahren 1992-2000 wurde für diese Statistik eine alternative Datenerhebung entwickelt. Erste Analysen hatten ergeben, dass die Untergrenze der großen Unternehmen auf 23 Millionen Euro angehoben werden konnte. Dadurch konnte die Anzahl großer Unternehmen, die voll mit Hilfe des Papierfragebogens erfasst werden, auf ungefähr 1.800 gesenkt werden. Weiterhin zeigte sich, dass es durch Nutzung von Registerdaten der Steuerbehörde möglich ist, die Daten für kleinere Unternehmungen vollständig aus Sekundärquellen zu erheben. Dazu werden für 200.000 kleinere Unternehmen Daten der Gesellschaftssteuer verwendet.

\section{Ausblick}

Das Statistische Amt der Niederlande wird sich aufgrund der Gesetzeslage künftig immer mehr der Erhebung von statistischer Information aus Registerdaten zuwenden müssen. Statistischer Output wird ganz oder teilweise aus Registerdaten geschätzt oder abgeleitet werden müssen. Weiterhin müssen derartige Quellen, wann immer möglich, als Hilfsinformation zur Entwicklung statistischer Modelle benutzt werden. Nur Information, die nicht aus Registern gewonnen werden kann, darf mit Hilfe von Primärdaten erhoben werden.

Neben der kontinuierlichen Erschließung von weiteren Sekundärquellen und dem effizienten Einsatz der primären Modi der Datenerhebung liegt die Herausforderung für das Statistische Amt der Niederlande vor allem in der Optimierung der Abstimmung zwischen sekundärer und primärer Datenerfassung. Dass dies sehr gut möglich ist, zeigen die Beispiele einer kombinierten primären und sekundären Datenerfassung bei den Structural Business Statistics und bei der Statistik der Finanzstrukturen von Unternehmen wie sie in den Niederlanden seit Mitte des letzten Jahrzehntes realisiert werden.

\section{Literatur}

Czaja, R., Blair, J. (2005) Designing Surveys: A guide to Decisions and Procedures. 2nd edn. Pine Forge Press, Thousand Oaks (Ca.)

Groves, R.M. (1989) Survey Errors and Survey Costs. Wiley, New York

Groves, R.M., Couper, M.P. (1998) Nonresponse in Household Interview Surveys. Wiley, New York

Groves, R.M., Fowler, F.J., Couper, M.P., Lepkowski, J.M., Singer, E., Tourangeau, R. (2004) Survey Methodology. Wiley, New York

Ministerie van de Vlaamse Gemeenschap (2001) Kwaliteitsrichtlijnen bij het uitvoeren van surveyonderzoek. Ministerie van de Vlaamse Gemeenschap, Administratie Planning en Statistiek, Brussel

ONS (2004) Guidelines for measuring statistical quality. Office for National Statistics, Londen

Statistics Canada (2003) Quality Guidelines. 4th edn. Statistics Canada, Ottawa

Statistics Finland (2002) Quality Guidelines for Official Statistics. Handbook 43b. Statistics Finland, Helsinki

Statistics Finland (2004) Use of Register and Administrative Data Sources for Statistical Purposes. Best Practices of Statistics Finland. Handbook 45. Statistics Finland, Helsinki

Thomas, M. (2005) Assessing the Quality of Administrative Data. Survey Methodology Bulletin, No. 56, September 2005, pp. 74-84 